\title{
Soliton solutions of Hirota equation and Hirota-Maccari system
}

\author{
M. M. El-Borai ${ }^{1}$, H. M. El-Owaidy ${ }^{2}$,Hamdy M. Ahmed ${ }^{3}$ and A. H. Arnous ${ }^{3, *}$ \\ ${ }^{1}$ Department of Mathematics, Faculty of science, Alexandria University, Egypt \\ ${ }^{2}$ Department of Mathematics, Faculty of science, Al-Azhar University, Egypt \\ ${ }^{3}$ Department of Engineering Mathematics and Physics, Higher Institute of Engineering, El Shorouk, Egypt
}

Received: 17 February 2016, Accepted: 14 March 2016

Published online: 21 July 2016.

\begin{abstract}
In this paper, the trial equation method is presented to seek the exact solutions of two nonlinear partial differential equations (NLPDEs), namely, the Hirota equation and the Hirota-Maccari system. The obtained solutions are solitary, topological, singular solitons and singular periodic waves. This method is powerful, effective and it can be extended to many NLPDEs.
\end{abstract}

Keywords: Solitons, exact solutions, trial equation method.

\section{Introduction}

In science, many important phenomena can be described by nonlinear partial differential equations. Seeking the exact solutions for these equations plays an important role in the study on the dynamics of those phenomena which appear in various scientific and engineering fields, such as solid state physics, fluid mechanics, chemical kinetics, plasma physics, population models, and nonlinear optics [1-17]. Many powerful methods have been proposed to obtain exact and approximated solutions of these models such as inverse scattering method [2,3], Hirota bilinear transformation [7], the F-expansion method [11,12,13], the modified simple equation method [10,11], the $\left(G^{\prime} / G\right)$-expansion method [12] and the trial equation method $[13,14]$. In the present work, we use the trial equation method for seeking the exact solutions for two important physical models, the Hirota equation and Hirota-Maccari System [16, 17]

$$
i u_{t}+u_{x x}+2|u|^{2} u+i \alpha u_{x x x}+6 i \alpha|u|^{2} u_{x}=0 .
$$

which defines the propagation of the femto-second soliton pulse in the single-mode fibers, where $u=u(x, t)$ is the complex amplitude of the slowly varying optical field, the subscripts $t$ and $x$, respectively, demonstrate the temporal and spatial partial derivatives and $\alpha$ is a small parameter and $u_{t}, u_{x x},|u|^{2} u, u_{x x x}$ and $|u|^{2} u_{x}$ indicate the linear evolution, the group velocity dispersion (GVD), self-phase modulation, third-order dispersion, and self-steepening, respectively.

$$
\begin{gathered}
i u_{t}+u_{x y}+i u_{x x x}+u v-i|u|^{2} u_{x}=0 \\
3 v_{x}+\left(|u|^{2}\right)_{y}=0
\end{gathered}
$$

where $u=u(x, y, t)$ and $v=v(x, y, t)$ represent the complex scalar field and the real scalar field, respectively, while $t$ represents the temporal variable $x, y$ are the independent spatial variables. 


\section{Description of the trial equation method}

In this subsection we outline the main steps of the trial equation method $[13,14]$ as following:

let us consider the nonlinear evolution equation (NLEE):

$$
F\left(u, u_{t}, u_{x}, u_{x x}, u_{x t}, \ldots\right)=0
$$

where $u=u(x, t)$ is an unknown function, $F$ is a polynomial in $u$ and its various partial derivatives $u_{t}, u_{x}$ with respect to $t, x$ respectively, in which the highest order derivatives and nonlinear terms are involved.

Using the traveling wave transformation

$$
u(x, t)=u(\xi), \xi=k(x-c t)
$$

where $k, c$ are constant to be determined later. Then Eq. (1) is reduced to a nonlinear ordinary differential equation of the form

$$
P\left(u, u^{\prime}, u^{\prime \prime}, \ldots\right)=0
$$

Step 1. Consider the trial equation

$$
\left(u^{\prime}\right)^{2}=F(u)=\sum_{l=0}^{s} a_{l} u^{l},
$$

where $a_{l},(l=0,1, \ldots, s)$ are constants to be determined. Substituting Eq. (4) and other derivative terms such as $u^{\prime \prime}$ or $u^{\prime \prime \prime}$ and so on into Eq.(3) yields a polynomial $G(u)$ of $u$. According to the balance principle we can determine the value of $s$. Setting the coefficients of $G(u)$ to zero, we get a system of algebraic equations. Solving this system, we shall determine $c, k$ and values of $a_{0}, a_{1}, \ldots, a_{s}$.

Step 2. Rewrite Eq. (4) by the integral form

$$
\pm\left(\xi-\xi_{0}\right)=\int \frac{1}{\sqrt{F(u)}} d u
$$

According to the complete discrimination system of the polynomial, we classify the roots of $F(u)$, and solve the integral equation (5). Thus, we obtain the exact solutions to Eq. (1).

\section{Applications}

In this section, we apply the trial equation method to solve the proposed models

\subsection{The Hirota equation}

The Hirota equation can be written as

$$
i u_{t}+u_{x x}+2|u|^{2} u+i \alpha u_{x x x}+6 i \alpha|u|^{2} u_{x}=0
$$

In this section, we employ the trial equation method to Eq. (6). To this end, we use the wave transformation

$$
u(x, t)=U(\xi) e^{i(p x+q t)}, \quad \xi=x+\omega t
$$


where $p, q, \omega$ are constants to be determined later. Now Eq. (6) is reduced to the following two ordinary differential equations:

$$
\begin{gathered}
\left(p^{3} \alpha-p^{2}-q\right) U(\xi)+(1-3 \alpha p) U^{\prime \prime}(\xi)+2(1-3 \alpha p) U^{3}(\xi)=0 \\
\left(\omega+2 p-3 \alpha p^{2}\right) U^{\prime}(\xi)+\alpha U^{\prime \prime \prime}(\xi)+6 \alpha U^{2}(\xi) U^{\prime}(\xi)=0 .
\end{gathered}
$$

From Eqs. (8) and (9), we can deduce that

$$
\omega=\frac{\alpha\left(p^{3} \alpha-p^{2}-q\right)}{1-3 \alpha p}+3 \alpha p^{2}-2 p
$$

and

$$
U^{\prime \prime}(\xi)+\left(\frac{p^{3} \alpha-p^{2}-q}{1-3 \alpha p}\right) U(\xi)+2 U^{3}(\xi)=0 .
$$

Balancing $U^{\prime \prime}$ with $U^{3}$ in Eq. (11), then we get $s=4$. Using the solution procedure of the trial equation method, we obtain the system of algebraic equations as follows:

$$
\begin{gathered}
\frac{a_{1}}{2}=0, \\
a_{2}+\frac{p^{3} \alpha-p^{2}-q}{1-3 \alpha p}=0, \\
\frac{3}{2} a_{3}=0, \\
2\left(a_{4}+1\right)=0 .
\end{gathered}
$$

By solving the above system of algebraic equations, we obtain the following results:

$$
a_{1}=0, \quad a_{2}=\frac{-p^{3} \alpha+p^{2}+q}{1-3 \alpha p}, \quad a_{3}=0, \quad a_{4}=-1
$$

By substituting these results into Eqs. (4) and (5), we get

$$
\pm\left(\xi-\xi_{0}\right)=\int \frac{1}{\sqrt{a_{0}+\frac{-p^{3} \alpha+p^{2}+q}{1-3 \alpha p} U^{2}-U^{4}}} d U .
$$

where $a_{0}$ is an arbitrary real constant. Now, we discuss two cases as following:

Case 1: If we set $a_{0}=0$ in Eq. (17) and integrating with resect to $U$, we get the following exact solution of Eq. (6)

$$
\begin{aligned}
& u(x, t)= \pm \sqrt{\frac{-p^{3} \alpha+p^{2}+q}{1-3 \alpha p}} \operatorname{sech}\left[\sqrt{\frac{-p^{3} \alpha+p^{2}+q}{1-3 \alpha p}}\left(x+\omega t-\xi_{0}\right)\right] e^{i(p x+q t)}, \\
& u(x, t)= \pm \sqrt{\frac{p^{3} \alpha-p^{2}-q}{1-3 \alpha p}} \operatorname{csch}\left[\sqrt{\frac{-p^{3} \alpha+p^{2}+q}{1-3 \alpha p}}\left(x+\omega t-\xi_{0}\right)\right] e^{i(p x+q t)} .
\end{aligned}
$$


These solutions are non-topological and singular soliton solutions which are valid for

$$
\begin{gathered}
\frac{-p^{3} \alpha+p^{2}+q}{1-3 \alpha p}>0 . \\
u(x, t)= \pm \sqrt{\frac{-p^{3} \alpha+p^{2}+q}{1-3 \alpha p}} \sec \left[\sqrt{\frac{p^{3} \alpha-p^{2}-q}{1-3 \alpha p}}\left(x+\omega t-\xi_{0}\right)\right] e^{i(p x+q t),} \\
u(x, t)=\mp \sqrt{\frac{-p^{3} \alpha+p^{2}+q}{1-3 \alpha p}} \csc \left[\sqrt{\frac{p^{3} \alpha-p^{2}-q}{1-3 \alpha p}}\left(x+\omega t-\xi_{0}\right)\right] e^{i(p x+q t)} .
\end{gathered}
$$

These solutions are couple of singular periodic solutions which are valid for

$$
\frac{-p^{3} \alpha+p^{2}+q}{1-3 \alpha p}<0
$$

Case 2: If we set $a_{0}=-\frac{\left(-\alpha p^{3}+p^{2}+q\right)^{2}}{4(1-3 \alpha p)^{2}}$ in Eq. (17) and integrating with resect to $U$, we get the following exact solution of Eq. (6)

$$
\begin{aligned}
& u(x, t)= \pm \sqrt{\frac{-p^{3} \alpha+p^{2}+q}{2(1-3 \alpha p)}} \tanh \left[\sqrt{\frac{p^{3} \alpha-p^{2}-q}{2(1-3 \alpha p)}}\left(x+\omega t-\xi_{0}\right)\right] e^{i(p x+q t)}, \\
& u(x, t)= \pm \sqrt{\frac{-p^{3} \alpha+p^{2}+q}{2(1-3 \alpha p)}} \operatorname{coth}\left[\sqrt{\frac{p^{3} \alpha-p^{2}-q}{2(1-3 \alpha p)}}\left(x+\omega t-\xi_{0}\right)\right] e^{i(p x+q t)} .
\end{aligned}
$$

These solutions are topological and singular 1-soliton solutions which are valid for

$$
\begin{gathered}
\frac{-p^{3} \alpha+p^{2}+q}{1-3 \alpha p}<0 . \\
u(x, t)= \pm \sqrt{\frac{p^{3} \alpha-p^{2}-q}{2(1-3 \alpha p)}} \tan \left[\sqrt{\frac{-p^{3} \alpha+p^{2}+q}{2(1-3 \alpha p)}}\left(x+\omega t-\xi_{0}\right)\right] e^{i(p x+q t)}, \\
u(x, t)=\mp \sqrt{\frac{p^{3} \alpha-p^{2}-q}{2(1-3 \alpha p)}} \cot \left[\sqrt{\frac{-p^{3} \alpha+p^{2}+q}{2(1-3 \alpha p)}}\left(x+\omega t-\xi_{0}\right)\right] e^{i(p x+q t)} .
\end{gathered}
$$

These solutions are singular periodic solutions which are valid for

$$
\frac{-p^{3} \alpha+p^{2}+q}{1-3 \alpha p}>0
$$

\subsection{The Hirota-Maccari system}

The Hirota-Maccari system can be written as

$$
\begin{aligned}
& i u_{t}+u_{x y}+i u_{x x x}+u v-i|u|^{2} u_{x}=0 \\
& 3 v_{x}+\left(|u|^{2}\right)_{y}=0
\end{aligned}
$$


In this section, we employ the trial equation method to Eq. (30). To this end, we use the wave transformation

$$
u(x, y, t)=U(\xi) e^{i(p x+q y+r t)}, \quad v(x, y, t)=V(\xi), \quad \xi=x+y+\omega t .
$$

where $p, q, r, \omega$ are constants to be determined later. Now Eq. (30) is reduced to the following two ordinary differential equations:

$$
\begin{aligned}
& 3(1-3 p) U^{\prime \prime}+3\left(p^{3}-p q-r\right) U+(3 p-1) U^{3}=0, \\
& V=-\frac{1}{3} U^{2}, \quad p \neq \frac{1}{3}
\end{aligned}
$$

Balancing $U^{\prime \prime}$ with $U^{3}$ in Eq. (32), then we get $s=4$. Using the solution procedure of the trial equation method, we obtain the system of algebraic equations as follows:

$$
\begin{gathered}
\left(6 a_{4}-1\right)(-(3 p-1))=0, \\
\frac{9}{2} a_{3}(1-3 p)=0, \\
3\left(a_{2}(1-3 p)+p^{3}-p q-r\right)=0, \\
\frac{3}{2} a_{1}(1-3 p)=0 .
\end{gathered}
$$

By solving the above system of algebraic equations, we obtain the following results:

$$
a_{1}=0, \quad a_{2}=\frac{p^{2}-p q-r}{3 p-1}, \quad a_{3}=0, \quad a_{4}=\frac{1}{6} .
$$

By substituting these results into Eqs. (4) and (5), we get

$$
\pm\left(\xi-\xi_{0}\right)=\int \frac{1}{\sqrt{a_{0}+\frac{p^{2}-p q-r}{3 p-1} U^{2}+\frac{1}{6} U^{4}}} d U .
$$

where $a_{0}$ is an arbitrary real constant. Now, we discuss two cases as following:

Case 1: If we set $a_{0}=0$ in Eq. (38) and integrating with resect to $U$, we get the following exact solution of Eq. (30)

$$
\begin{gathered}
u(x, y, t)= \pm \sqrt{\frac{6\left(-p^{2}+p q+r\right)}{3 p-1}} \operatorname{sech}\left[\sqrt{\frac{p^{2}-p q-r}{3 p-1}}\left(x+y+\omega t-\xi_{0}\right)\right] e^{i(p x+q y+r t)}, \\
v(x, y, t)=\frac{2\left(p^{2}-p q-r\right)}{3 p-1} \operatorname{sech}^{2}\left[\sqrt{\frac{p^{2}-p q-r}{3 p-1}}\left(x+y+\omega t-\xi_{0}\right)\right], \\
u(x, y, t)= \pm \sqrt{\frac{6\left(p^{2}-p q-r\right)}{3 p-1}} \operatorname{csch}\left[\sqrt{\frac{p^{2}-p q-r}{3 p-1}}\left(x+y+\omega t-\xi_{0}\right)\right] e^{i(p x+q y+r t)},
\end{gathered}
$$




$$
v(x, y, t)=\frac{2\left(-p^{2}+p q+r\right)}{3 p-1} \operatorname{csch}^{2}\left[\sqrt{\frac{p^{2}-p q-r}{3 p-1}}\left(x+y+\omega t-\xi_{0}\right)\right] .
$$

These solutions are non-topological and singular soliton solutions which are valid for

$$
\begin{gathered}
\frac{p^{2}-p q-r}{3 p-1}>0 . \\
u(x, y, t)=\mp \sqrt{\frac{6\left(-p^{2}+p q+r\right)}{3 p-1}} \sec \left[\sqrt{\frac{-p^{2}+p q+r}{3 p-1}}\left(x+y+\omega t-\xi_{0}\right)\right] e^{i(p x+q y+r t),} \\
v(x, y, t)=\frac{2\left(p^{2}-p q-r\right)}{3 p-1} \sec ^{2}\left[\sqrt{\frac{-p^{2}+p q+r}{3 p-1}}\left(x+y+\omega t-\xi_{0}\right)\right], \\
u(x, y, t)= \pm \sqrt{\frac{6\left(-p^{2}+p q+r\right)}{3 p-1}} \csc ^{2}\left[\sqrt{\left.\frac{-p^{2}+p q+r}{3 p-1}\left(x+y+\omega t-\xi_{0}\right)\right] e^{i(p x+q y+r t)},}\right. \\
v(x, y, t)=\frac{2\left(p^{2}-p q-r\right)}{3 p-1} \csc ^{2}\left[\sqrt{\frac{-p^{2}+p q+r}{3 p-1}}\left(x+y+\omega t-\xi_{0}\right)\right] .
\end{gathered}
$$

These solutions are couple of singular periodic solutions which are valid for

$$
\frac{p^{2}-p q-r}{3 p-1}<0
$$

Case 2: If we set $a_{0}=\frac{3\left(p^{3}-p q-r\right)^{2}}{2(3 p-1)^{2}}$ in Eq. (38) and integrating with resect to $U$, we get the following exact solution of Eq. (30)

$$
\begin{gathered}
u(x, y, t)= \pm \sqrt{\frac{3\left(-p^{2}+p q+r\right)}{3 p-1}} \tanh \left[\sqrt{\frac{-p^{2}+p q+r}{2(3 p-1)}}\left(x+y+\omega t-\xi_{0}\right)\right] e^{i(p x+q y+r t)}, \\
v(x, y, t)=\frac{\left(p^{2}-p q-r\right)}{3 p-1} \tanh ^{2}\left[\sqrt{\frac{-p^{2}+p q+r}{2(3 p-1)}}\left(x+y+\omega t-\xi_{0}\right)\right], \\
u(x, y, t)= \pm \sqrt{\frac{3\left(-p^{2}+p q+r\right)}{3 p-1}} \operatorname{coth}\left[\sqrt{\frac{-p^{2}+p q+r}{2(3 p-1)}}\left(x+y+\omega t-\xi_{0}\right)\right] e^{i(p x+q y+r t),} \\
v(x, y, t)=\frac{\left(p^{2}-p q-r\right)}{3 p-1} \operatorname{coth}^{2}\left[\sqrt{\frac{-p^{2}+p q+r}{2(3 p-1)}}\left(x+y+\omega t-\xi_{0}\right)\right] .
\end{gathered}
$$

These solutions are topological and singular 1-soliton solutions which are valid for

$$
\frac{p^{2}-p q-r}{3 p-1}<0
$$




$$
\begin{gathered}
u(x, y, t)= \pm \sqrt{\frac{3\left(p^{2}-p q-r\right)}{3 p-1}} \tan \left[\sqrt{\frac{p^{2}-p q-r}{2(3 p-1)}}\left(x+y+\omega t-\xi_{0}\right)\right] e^{i(p x+q y+r t),} \\
v(x, y, t)=\frac{\left(-p^{2}+p q+r\right)}{3 p-1} \tan ^{2}\left[\sqrt{\frac{p^{2}-p q-r}{2(3 p-1)}}\left(x+y+\omega t-\xi_{0}\right)\right], \\
u(x, y, t)=\mp \sqrt{\frac{3\left(p^{2}-p q-r\right)}{3 p-1}} \cot \left[\sqrt{\frac{p^{2}-p q-r}{2(3 p-1)}}\left(x+y+\omega t-\xi_{0}\right)\right] e^{i(p x+q y+r t),} \\
v(x, y, t)=\frac{\left(-p^{2}+p q+r\right)}{3 p-1} \cot ^{2}\left[\sqrt{\frac{p^{2}-p q-r}{2(3 p-1)}}\left(x+y+\omega t-\xi_{0}\right)\right] .
\end{gathered}
$$

These solutions are singular periodic solutions which are valid for

$$
\frac{p^{2}-p q-r}{3 p-1}>0
$$

\section{Conclusions}

In this paper, we successfully applied the proposed method to solve the Hirota equation and the Hirota-Maccari system. As a result of this application different types of solitons are obtained such as solitary, topological, singular soliton, singular periodic waves etc. The constraint conditions for the existence of these solutions are given.

\section{Competing interests}

The authors declare that they have no competing interests.

\section{Authors' Contributions}

All authors have contributed to all parts of the article. All authors read and approved the final manuscript.

\section{References}

[1] P. Gray and S. Scott, Chemical oscillations and instabilities, Clarendon, Oxford, 1990.

[2] M. J. Ablowitz and P. A. Clarkson, Soliton, Nonlinear evolution equations and inverse scattering transform, Cambridg University Press, New York, 1991.

[3] V. O. Vakhnenko, E. J. Parkes and A. J. Morrison, A Backlund transformation and the inverse scattering transform method for the generalized Vakhnenko equation, Chaos, Solitons and Fractals, 17 (2003), 683.

[4] Mahmoud M. El-Borai, Exact solutions for some nonlinear fractional parabolic fractional partial differential equations, Journal of Applied Mathematics and Computation 206 (2008,) 141-153.

[5] Mahmoud M. El-Borai, Afaf A. Zaghrout and Amal L. Elshaer, Exact solutions for nonlinear partial differential equations by using the extended multiple Riccati equations expansion method, Inter. J. of Research and Reviews in Applied Sciences, 9(3) (2011), 370-381. 
[6] E. M. E. Zayed and A.H. Arnous, DNA dynamics studied using the homogeneous balance method, Chinese Physics Letters, 29(8) (2012), 080203.

[7] R. Hirota, Direct method of finding exact solutions of nonlinear evolution equations, in: R. Bulloygh, P. Coudrey (Eds.), Bäcklund transformation, Springer, Berlin, (1980).

[8] M. L. Wang, X. Li, Applications of F-expansion to periodic wave solutions for a new Hamiltonian amplitude equation. Chaos Solit. Fract., 24 (2005), 1257-1268.

[9] D. S. Wang, H. Q. Zhang, Further improved F-expansion method and new exact solutions of Konopelchenko-Dubrovsky equations. Chaos Solit. Fract., 25 (2005) 601-610.

[10] M. A. M. Jawad, M. D. Petkovic and A. Biswas, Modified simple equation method for nonlinear evolution equations, Appl. Math. Comput., 217 (2010), 869-877.

[11] E. M. E. Zayed and A.H. Arnous, Exact solutions of the nonlinear ZK-MEW and the Potential YTSF equations using the modified simple equation method, AIP Conf. Proc., 1479 (2012), 2044-2048.

[12] M. L. Wang and X. Li , J. Zhang, The $\left(G^{\prime} / G\right)$-expansion method and traveling wave solutions of nonlinear evolution equations in mathematical physics, Phys. Lett. A., 372 (2008) 417-423.

[13] C.S. Liu, Trial equationmethod to nonlinear evolution equations with rank inhomogeneous: mathematical discussions and its applications, Commun. Theor. Phys. 45 (2006), 219-223.

[14] M. Mirzazadeh, A. H. Arnous, M. F. Mahmood, E. Zerrad and A. Biswas, Soliton solutions to resonant nonlinear schrödinger's equation with time-dependent coefficients by trial solution approach, Nonlinear Dynamics, 81(8) (2015), 277-282.

[15] M. Mirzazadeh, A. H. Arnous and M. Eslami, Dark optical solitons of Biswas-Milovic equation with dual-power law nonlinearity, The European Physical Journal Plus, 130(4) (2015), 1-7.

[16] S. T. Demiray and Y. Pandir, H. Bulut, All exact travelling wave solutions of Hirota equation and Hirota-Maccari system, Optik, 127(4) (2016), 1848-1859.

[17] M. Eslami, M. A. Mirzazadeh, A. Neirameh, New exact wave solutions for Hirota equation, Pramana J. of Physics, 84(1) (2015), $3-8$. 